\title{
A CONVERGENCE PROBLEM CONNECTED WITH CONTINUED FRACTIONS
}

\author{
GERHARD LARCHER
}

(Communicated by Larry J. Goldstein)

\begin{abstract}
The set $Z_{\alpha}:=\left\{\beta \mid \lim _{n \rightarrow \infty}\left\|q_{n} \beta\right\|=0\right\}$ is considered, where $\left(q_{n}\right)_{n \in \mathbf{N}}$ is the sequence of best approximation denominators of $\alpha$, and it is explicitly determined for $\alpha$ with bounded continued fraction coefficients.
\end{abstract}

Introduction. Let the irrational number $\alpha$ have continued fraction expansion $\alpha=\left[a_{0} ; a_{1}, a_{2}, a_{3}, \ldots\right]$ and let $q_{-1}=0,1=q_{0} \leq q_{1}<q_{2}<\cdots$ with $q_{i+1}=$ $a_{i+1} q_{i}+q_{i-1}$ be the best approximation denominators of $\alpha$. By Theorem 4.3 in [2] it follows, that $\left\{q_{n} \beta\right\}_{n \in \mathrm{N}}$ (where $\{x\}:=x-[x]$ denotes the fractional part of $x$ ) is uniformly distributed modulo one for almost all $\beta \in \mathbf{R}$ in the sense of Lebesgue measure. Clearly the sequence is not uniformly distributed for all $\beta$, since if we take $\beta=m \alpha+n$ with $m, n \in \mathbf{Z}$, then $\lim _{n \rightarrow \infty}\left\|q_{n} \beta\right\|=0$ where $\|x\|$ denotes the distance from $x$ to the nearest integer.

We ask now for the set $Z_{\alpha}:=\left\{\beta \in \mathbf{R} \mid \lim _{n \rightarrow \infty}\left\|q_{n} \beta\right\|=0\right\}$. The problem of determining this set also is of some importance in some problems of automata theory [3]. We will show

THEOREM 1. If $\alpha$ has bounded continued fraction coefficients, then

$$
\lim _{n \rightarrow \infty}\left\|q_{n} \beta\right\|=0
$$

if and only if $\beta=m \alpha+n$ with $m, n \in \mathbf{Z}$.

This in general is not true if $\alpha$ does not have bounded continued fraction coefficients, for we can show

THEOREM 2. There are $\alpha \in \mathbf{R}$ and $\beta \neq m \alpha+n$ for all $m, n \in \mathbf{Z}$ with

$$
\lim _{n \rightarrow \infty}\left\|q_{n} \beta\right\|=0 \text {. }
$$

PROOFS. For the proof of Theorem 1 we need two lemmata. In these two lemmata let $\alpha$ be a fixed real number and the $q_{i}$ as above.

LEMMA 1. Let $a_{i} \leq K-1$ for all $i \in \mathbf{N}$ and let $n, p_{n}, p_{n+1} \in \mathbf{N}$ be given, then there exists at most one $\beta \in[0,1)$ with

and

$$
\left|q_{n} \beta-p_{n}\right| \leq \frac{1}{4 K}, \quad\left|q_{n+1} \beta-p_{n+1}\right| \leq \frac{1}{4 K}
$$

$$
\left\|q_{m} \beta\right\| \leq \frac{1}{4 K} \quad \text { for all } m \geq n .
$$

Received by the editors October 14, 1986 and, in revised form, June 23, 1987.

1980 Mathematics Subject Classification (1985 Revision). Primary 10A32, 10F20.

Key words and phrases. Continued fractions. 
PROOF. For any $\beta$ which fulfills our conditions, we have

$$
q_{n+2} \beta \in I_{n+2}:=\left(p_{n+1} \cdot \frac{q_{n+2}}{q_{n+1}}-\frac{q_{n+2} / q_{n+1}}{4 K}, p_{n+1} \cdot \frac{q_{n+2}}{q_{n+1}}+\frac{q_{n+2} / q_{n+1}}{4 K}\right) .
$$

The length of $I_{n+2}$ is $\leq \frac{1}{2}$ and because of $\left\|q_{n+2} \beta\right\| \leq 1 / 4 K$, there is, independent of $\beta$, exactly one $p_{n+2}$ with $\left|q_{n+2} \beta-p_{n+2}\right| \leq 1 / 4 K$. Going on this way, we see, that independent of $\beta$, by $n, p_{n}$ and $p_{n+1}$ all further values $p_{n+2}, p_{n+3}, \ldots$ are determined, for which $\left|q_{m} \beta-p_{m}\right| \leq 1 / 4 K$. Therefore $\beta$ is unique, because $\beta=\lim _{m \rightarrow \infty}\left(p_{m} / q_{m}\right)$.

LEMMA 2. Let $\beta, p_{n}$ and $p_{n+1}$ be such that $\left|q_{n} \beta-p_{n}\right|<1 / 8 K,\left|q_{n+1} \beta-p_{n+1}\right|$ $<1 / 8 K, p_{n} / q_{n} \neq p_{n+1} / q_{n+1}, \operatorname{sgn}\left(q_{n} \beta-p_{n}\right)=-\operatorname{sgn}\left(q_{n+1} \beta-p_{n+1}\right)$ and $\left\|q_{m} \beta\right\|<1 / 4 K$ for $m \geq n$; if we define $\lambda_{n}:=\left[0 ; a_{n}, a_{n+1}, \ldots\right]$ and $\alpha_{0}:=$ $\left(p_{n+1}+\lambda_{n+1} p_{n}\right) /\left(q_{n+1}+\lambda_{n+1} q_{n}\right)$, then $\beta=\alpha_{0}$.

ProOF. We show $\left|q_{n} \alpha_{0}-p_{n}\right|<1 / 4 K,\left|q_{n+1} \alpha_{0}-p_{n+1}\right|<1 / 4 K$ and $\left\|q_{m} \alpha_{0}\right\|<$ $1 / 4 K$ for $m \geq n$ and then by Lemma 1 the result follows. We have

$$
\begin{aligned}
\left|q_{n} \cdot \frac{p_{n+1}+\lambda_{n+1} p_{n}}{q_{n+1}+\lambda_{n+1} q_{n}}-p_{n}\right| & =\left|\frac{q_{n} p_{n+1}-q_{n+1} p_{n}}{q_{n+1}+\lambda_{n+1} q_{n}}\right| \leq\left|q_{n} \cdot \frac{p_{n+1}}{q_{n+1}}-p_{n}\right| \\
& \leq\left|q_{n} \beta-p_{n}\right|+\left|q_{n} \cdot \frac{1}{8 K q_{n+1}}\right| \leq \frac{1}{4 K}
\end{aligned}
$$

and

$$
\left|q_{n+1} \cdot \frac{p_{n+1}+\lambda_{n+1} p_{n}}{q_{n+1}+\lambda_{n+1} q_{n}}-p_{n+1}\right|=\left|\lambda_{n+1} \cdot \frac{p_{n} q_{n+1}-q_{n} p_{n+1}}{q_{n+1}+\lambda_{n+1} q_{n}}\right| \leq \frac{1}{4 K} .
$$

Now we consider in the plane the lattice $\Gamma$ produced by the vectors $\left(q_{n}, p_{n}\right)$ and $\left(q_{n+1}, p_{n+1}\right)$ which are independent, and the line

$$
g: \quad y=\frac{p_{n+1}+\lambda_{n+1} p_{n}}{q_{n+1}+\lambda_{n+1} q_{n}} \cdot x .
$$

This lattice is isomorphic to the lattice $\Gamma^{\prime}$ produced by $(0,1),(1,0)$ and the line $g^{\prime}: y=\lambda_{n+1} \cdot x$.

Now we consider the Klein-model of the development of $\lambda_{n+1}$ to continued fractions as it is explained for example in $[\mathbf{1}] . \Gamma^{\prime}$ is the lattice for this development.

By observing the same operations in $\Gamma$ as in $\Gamma^{\prime}$ according to this development we see that

$$
\left\|q_{m} \alpha_{0}\right\| \leq \max \left(\left\|q_{n} \alpha_{0}\right\|,\left\|q_{n+1} \alpha_{0}\right\|\right)<\frac{1}{4 K} \quad \text { for all } m \geq n
$$

(See Figure 1 and Figure 2.)

For example we have (see Figure 3) $\left\|q_{n+3} \alpha_{0}\right\|=\left|R_{2} T_{2}\right|$ and since $\left|P_{2} S_{2}^{\prime}\right| \leq$ $\max \left(\left|P_{-1} 0\right|,\left|P_{0} S_{0}^{\prime}\right|\right)$ we have $\left|R_{2} S_{2}\right| \leq \max \left(\left|R_{-1} 0\right|,\left|R_{0} S_{0}\right|\right)$ and therefore

$$
\left|R_{2} T_{2}\right| \leq \max \left(\left|R_{-1} T_{0}\right|,\left|R_{0} T_{1}\right|\right)=\max \left(\left\|q_{n} \alpha_{0}\right\|,\left\|q_{n+1} \alpha_{0}\right\|\right),
$$

and the result follows.

PROOF OF THEOREM 1. Let $\lim _{n}\left\|q_{n} \beta\right\|=0$. Of course $\beta$ must be irrational or an integer. So let $\beta$ be irrational. We define by $p_{n}$ the integer lying next to $q_{n} \beta$, and $N_{0}$ to be such, that $\left\|q_{n} \beta\right\|<1 / 8 K$ for $n \geq N_{0}$. 


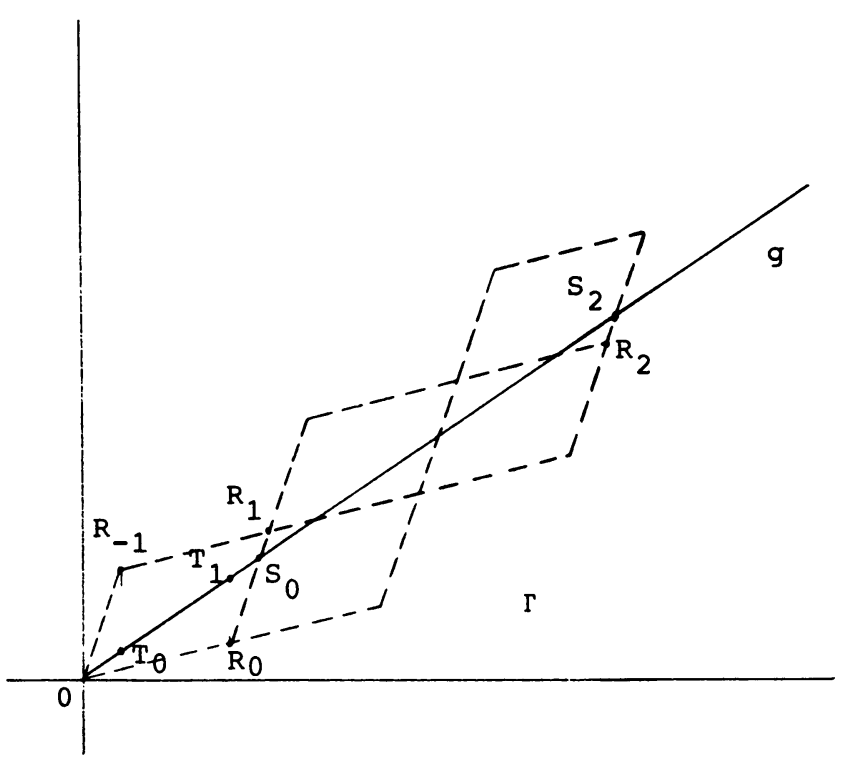

\section{FIGURE 1}

We show, that there is a $n \geq N_{0}$ such, that $\operatorname{sgn}\left(q_{n} \beta-p_{n}\right)=-\operatorname{sgn}\left(q_{n+1} \beta-p_{n+1}\right)$. Since $\beta \notin \mathbf{Q}$, from this it follows, that $p_{n} / q_{n} \neq p_{n+1} / q_{n+1}$.

If for example $q_{n} \beta-p_{n}>0$ for all $n \geq N_{0}$, then for all $n \geq N_{0}+1$ we would have $q_{n+1} \beta=a_{n} q_{n} \beta+q_{n-1} \beta$. Further $q_{n} \beta=p_{n}+\varepsilon_{0}, q_{n-1} \beta=p_{n-1}+\varepsilon_{1}$ with $0<\varepsilon_{i}<1 / 8 K$ and therefore $q_{n+1} \beta=a_{n} p_{n}+p_{n-1}+a_{n} \varepsilon_{0}+\varepsilon_{1}$ with $0<a_{n} \varepsilon_{0}+\varepsilon_{1}<$ $1 / 8+1 / 8 K \leq 1 / 4$ and therefore $\left\|q_{n+1} \beta\right\|=a_{n} \varepsilon_{0}+\varepsilon_{1} \geq\left\|q_{n} \beta\right\|$ for all $n \geq N_{0}+1$ and this is a contradiction since $\left\|q_{N_{0}+1} \beta\right\| \neq 0$. So by Lemma 2

$$
\beta=\frac{p_{n+1}+\lambda_{n+1} p_{n}}{q_{n+1}+\lambda_{n+1} q_{n}} .
$$

If we define $Q_{i}$ such, that $\left|q_{i} \alpha-Q_{i}\right| \leq 1 / q_{i+1}$ for all $i$, then (see [4]).

$$
\lambda_{n+1}=\left[0 ; a_{n+1}, a_{n+2}, \ldots\right]=-\frac{Q_{n+1}-\alpha q_{n+1}}{Q_{n}-\alpha q_{n}}
$$

and therefore

$$
\beta=\frac{\left(p_{n+1} Q_{n}-Q_{n+1} p_{n}\right)+\alpha\left(q_{n+1} p_{n}-q_{n} p_{n+1}\right)}{\left(q_{n+1} Q_{n}-Q_{n+1} q_{n}\right)}=a \alpha+b \quad \text { with } a, b \in \mathbf{Z}
$$

because $\left|q_{n+1} Q_{n}-Q_{n+1} q_{n}\right|=1$, and the theorem is proved.

PROOF OF THEOREM 2. Let $\gamma$ and $\delta$ irrational be such that

$$
\delta \neq(a \gamma+b) /(c \gamma+d) \quad \text { for all } a, b, c, d \in \mathbf{Z}
$$

and such that $1, \gamma, \delta$ are linear independent over $\mathbf{Q}$. Therefore not both are of the form $e \alpha+f$ with $e, f \in \mathbf{Z}$. We define now a real number $\alpha$ by its partial quotients $a_{0}, a_{1}, \ldots$ such that for the best approximation denominators $q_{n}$ of $\alpha$ we have $\lim _{n \rightarrow \infty}\left\|q_{n} \gamma\right\|=0$ and $\lim _{n \rightarrow \infty}\left\|q_{n} \delta\right\|=0$.

Let $a_{0}=0, q_{-1}=0, q_{0}=1$ and assume that $a_{1}, a_{2}, \ldots, a_{n}$ and therefore $q_{1}, q_{2}, \ldots, q_{n}$ are defined. 


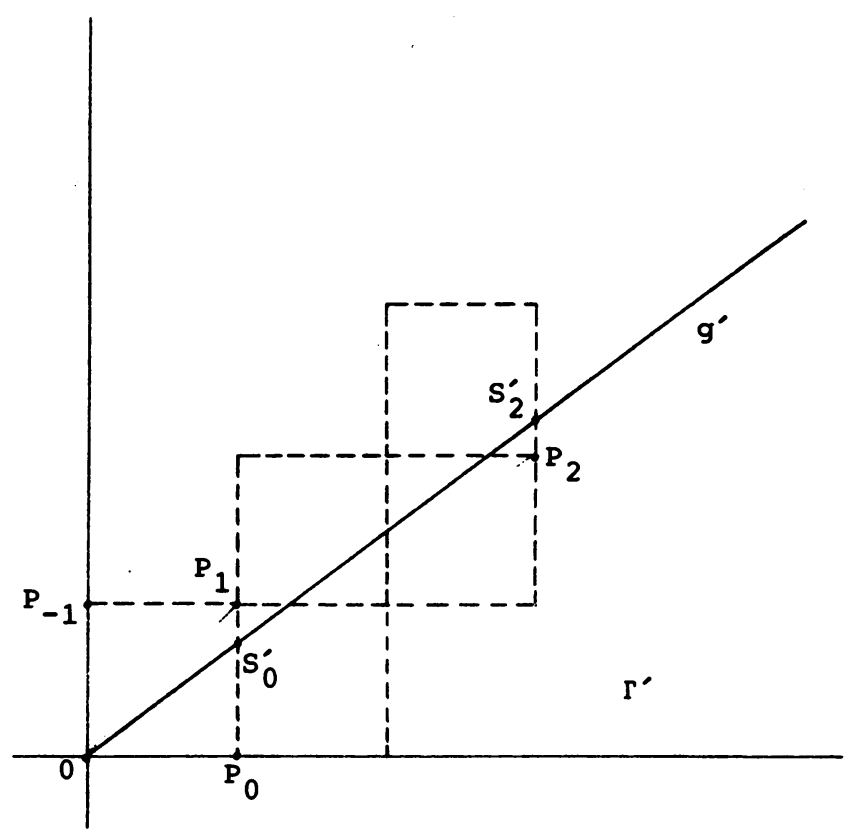

FIGURE 2

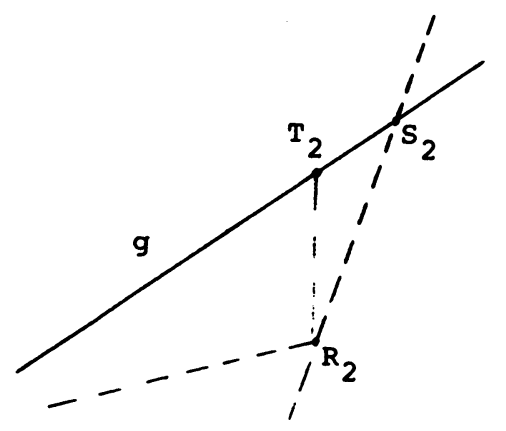

FIGURE 3

$1, q_{n} \gamma$ and $q_{n} \delta$ are linearly independent over $\mathbf{Q}$ and therefore the sequence $\left(\left\{k q_{n} \gamma\right\},\left\{k q_{n} \delta\right\}\right)_{k \in N}$ is dense in the unit square. Therefore we can choose an integer $a_{n+1}$ such that

$$
\max \left(\left\|a_{n+1} q_{n} \gamma+q_{n-1} \gamma\right\|,\left\|a_{n+1} q_{n} \delta+q_{n-1} \delta\right\|\right) \leq 1 / n .
$$

Then with $q_{n+1}=a_{n+1} q_{n}+q_{n-1}$ we have $\lim _{n \rightarrow \infty}\left\|q_{n} \beta\right\|=0$ for $\beta=\gamma$ and for $\beta=\delta$ and the theorem is proved. 


\section{REFERENCES}

1. A. J. Brentjes, Multi-dimensional continued fraction algorithms, Mathematisch Centrum, Amsterdam, 1981.

2. L. Kuipers, and H. Niederreiter, Uniform distribution of sequences, Wiley, New York, 1974.

3. C. Mauduit, Uniform distribution of $\alpha$-scale automata-sequences, Marseille, 1986 (to appear).

4. O. Perron, Die Lehre von den Kettenbrüchen, Teubner, Stuttgart, 1954.

Institut Für MAthematik, Hellbrunnerstrasse 34, A-5020 SAlzbURG, AUStria 